\section{INVEST NOW IN AN ISA}

money4dentists advise that savers can benefit greatly from making use of their annual Individual Savings Accounts (ISA) allowance. With most of the tax year still to come, it is desirable to increase investment in your ISA rapidly - particularly as the annual allowance of up to $£ 10,680$ cannot be carried over into the tax year beginning 5 April 2012.

The primary benefit of ISAs is that they are very tax efficient. Business owners (including dental practitioners) also do not have to give details about their ISAs on their tax returns. For older investors, it is worth noting that ISA income has no effect on age related allowances for the over-65s.

Tax is not the only reason to choose an ISA, however. Their flexibility allows savers to access them whenever they like, and they can be opened with a lump sum of just $€ 1$. Investors are also allowed to switch providers and from fund to fund if their ISA performs poorly or their current ISA providers charges are too high.

For more adventurous depositors, potentially greater profits make stocks and shares ISAs a tempting option (sales reached £3.9 billion in 2010). Diversifying share portfolios by investing in multiple funds is another useful feature.

A plethora of new ISA accounts, offering excellent interest rates, routinely appear during the latter part of the tax year. Specialist Independent Financial Advisers money4dentists can advise you on which ISA is right for you.

Reader response number 61

\section{UNPARALLELED PERFORMANCE}

The DUO Plus symbolises Castellini’s reputation for excellence, capturing its design philosophy that combines flexibility and ergonomics with outstanding performance.

Dental professionals who demand the best need look no further than the DUO Plus from Castellini, which blends innovative solutions, unrivalled hygiene systems and perfectly integrated instruments together to provide unparalleled levels of performance. Attributes include:

- Full operating freedom with or without assistant

- Adaptable to the operating style of the dentist

- Dentist's module has side-mounted control panel and can house up to five instruments, with an adjustable tray holder

- Assistant's module is placed on an articulated arm with over $30 \mathrm{~cm}$ vertical adjustment possible

- Instruments arranged for convenience

- Patient chair movement managed directly or via memory programes; smooth movement with hydraulic mechanism

- Pneumatic headrest pivots on three axes allowing precise positioning of patient's head

- Manoeuvrability of assistant's module for a wide range of working positions

- Instrument module is vertically adjustable so that optimal ergonomics is achieved in whatever situation

- Chair base foot switch can temporarily suspend suction.

The DUO Plus from Castellini provides the highest quality and performance without any compromise. Reader response number 62

\title{
DELIVERING SATISFACTION ALL ROUND
}

Sirona Dental Systems are pleased to announce the benefits that the CEREC AC Bluecam CAD/CAM system in conjunction with the Biogeneric software offers to the dental practice.

CEREC Biogeneric revolutionises occlusal surface design. It is the first restoration method which metrically analyses the patient's individual dentition status as the basis for computing the occlusal morphology. This patented scientific procedure is the passport to natural, made-to-measure restorations founded on precise metric principles. What is more, this method is extremely simple. With a single mouse click the user is able to create crowns, veneers, inlays and onlays, as well as anatomically sized bridges with up to four units.

CEREC significantly improves the quality of treatment for patients, as well as enhancing the efficiency of the dental practice. CEREC not only saves time for you and your patients, but also drastically reduces your laboratory bills which are a costly monthly expense.

Sirona Dental Systems Ltd UK's mission is to deliver satisfaction to the dentist using tried and tested training methods supported by CEREC Specialists who are dedicated to your success. Reader response number 63

\section{GENTLE, DURABLE, EFFECTIVE}

Curaprox have developed a gentle and easy-to-use range of durable, ultrafine interdental brushes designed to make interproximal cleaning that bit easier, encouraging patients to take responsibility for the health of their gums.

A brush that fails to completely fill the interdental space will not perform its job efficiently. As a result, Curaprox has developed a range of colour-coded inter-changeable brush heads of differing sizes that can be fitted onto any Curaprox brush handle, making them portable and easy to distinguish.

Gentle on gums, thanks to their soft, flexible filaments, each inter-dental brush is made of a patented nickel free alloy called CURAL, which lasts up to five times longer than other brushes, whilst also offering an ultrafine core width.

With 30 years of experience in the field of oral care, Curaprox is able to offer practitioners a wide array of innovative products designed to assist patients in taking care of their oral health.

Reader response number 64 